\title{
CANNULATED SCREW FIXATION FOR DISLOCATED FRACTURES OF THE CALCANEUS
}

\author{
Stoyan Ivanov \\ St. Anna University Hospital, Varna
}

\begin{abstract}
INTRODUCTION: Fractures of the calcaneus still remain some of the most challenging injuries that an orthopedic surgeon should manage. The difficulties about these fractures arise from the specific anatomy of the region, from the unique shape and morphology of the bone, and from the mechanism of the injury (in most cases high energy trauma). Despite the progress of the medical implants that can be used for fracture fixation in the last decade there are a lot of controversies, regarding the best option.
\end{abstract}

AIM: The aim of the study is to present our experience with the use of cannulated screws for fixation of dislocated fractures of os calcis.

MATERIALS AND METHODS: Twenty patients with dislocated fractures of os calcis using cannulated screws for fixation were operated on for a period of two years (2017-2018). The classifications used in the study included: the Essex-Lopresti $x$-ray classification modified by Stulik and Stehlik and the CT classification of Sanders. Two of the patients were with dislocated extraarticular fractures of tuber calcanei, three patients with "extreme beak" type fractures, the remaining 15 patients were with intraarticular fractures with more than $2 \mathrm{~mm}$ step off in the subtalar joint: 9 patients with tongue type and 5 patients with joint depression type. Gender distribution: 18 men, 2 women.

RESULTS: Evaluation of the results was made by using the Maryland Foot Score. Satisfaction from the treatment was graded using the Visual Analogue Scale (VAS) score (mean 7,5). The reduction is evaluated by measurement of the angle of Gissane, Bohler and Preis pre- and postoperatively. Assessment of the calcaneal height, length and width was initiated on lateral, axial and Broden views.

CONCLUSION: Fixation of displaced calcaneal fractures by cannulated screw is effective and reliable method. These screws are introduced by stab incisions which leads to minimal soft tissue dissection in such complex area of the hindfoot. It is an enormous advantage for fracture healing and reduces the percentage of possible complications.

Keywords: cannulated screw, calcaneus fractures, fixation

Address for correspondence:

Stoyan Ivanov

St. Anna University Hospital

100 Tzar Osvoboditel Blvd

9002 Varna

e-mail:ton_ivanov@abv.bg

Received: May 27, 2019

Accepted: June 16, 2019

\section{INTRODUCTION}

Calcaneal fractures are the most common tarsal bone fractures (about 60\%). These fractures can be intraarticular or extraarticular as intraarticular fractures comprise approximately $75 \%$ of all calcaneus fractures. Fractures of the calcaneus are complex injuries often associated with fractures of the spine, thorax or extremities. The bone itself has spe- 
cific anatomy with thin soft tissue envelope, which further complicates surgical treatment (1). In most cases these fractures are results of high energy trauma - fall from heights or due to traffic accident. Calcaneal fractures have enormous social and economic impact due to the often prolonged period of hospitalization, long rehabilitation and difficult recovery which may exceed more than six months. Also most of these injuries affect young or middle-aged active people.

Os calcis is the largest tarsal bone and forms the back of the longitudinal arch of the foot. It consists of dense spongy bone covered with a thin cortical bone, with the exception of the tuber calcanei, which has a thickened cortex. The bone is irregular in shape; it has four articulating surfaces that provide contact with the surrounding tarsal bones. Deformity of the bone after fracture include: loss of height, reduce length, increase wide, comminution of the lateral wall, subtalar joint incongruence. All these pathoanatomical changes may lead to subtalar joint arthritis, anterior ankle impingement, peroneal tendon impingement, calcaneo-fibular impingement, heel varus, limb length discrepancy, reduced gastrocnemius power.

Calcaneus fractures may be treated with a variety of methods, surgical approaches and different implants. There is still no consensus regarding the best surgical approach or method of fixation, even more, in a lot of cases the results are still unsatisfactory (2).

Non-operative treatment of these fractures leads to subtalar arthrosis, stiffness, difficulties in shoe wearing due to disturbed morphology of the bone, limping and pain especially on uneven ground. During the years different operative techniques have been used from Kirschner wire fixation, pins, staples, external fixators to extensile open approaches/ dual approaches and different plates for fixation (3). The percent of postoperative wound complications of open reduction vary from $10 \%$ to $20 \%$ and high risk regarding soft tissue complications are associated with patients with diabetics, vascular diseases and smokers.

Understanding and evaluation of the fracture mechanism is essential for minimally invasive reduction and fixation of calcaneal fractures. Detailed knowledge and assessment of the direction of primary and secondary fracture lines is mandatory in preoperative planning therefore X-ray examination (lateral, axial, a-p view) and CT scan with 3D reconstruction should be performed (4). In the last years vast majority of authors have paid special attention to the soft tissue component of the fracture in order to achieve higher postoperative results.

\section{AIM}

The aim of the study is to present our experience with the use of cannulated screws for fixation of dislocated fractures of os calcis.

\section{MATERIALS AND METHODS}

We have performed a retrospective study which included 20 patients operated in the Department of Orthopedics and Traumatology at Saint Anna University Hospital Varna for a period of two years (20172018). Two of the patients were with dislocated extraarticular fractures of tuber calcanei, three patients with "extreme beak" type fractures, the remaining 15 patients were with intraarticular fractures with more than $2 \mathrm{~mm}$ step off in the subtalar joint: 9 patients with tongue type, and 5 patients with joint depression type. The gender distribution was: 18 men, 2 women. The mean age was 45.5 years. Classifications that were used in the study included: the EssexLopresti x-ray classification modified by Stulik and Stehlik (5) and the CT classification of Sanders (6).

The goal of the operative treatment included: anatomic reduction of joint surface, restoration of normal bone morphology (correction of height Bohler angle, length, width and position of bone), reduction of wound problems and complications, restoration of biomechanics of subtalar joint (7). Depending on the fracture type and dislocation, we used different types of cannulated screw for fracture fixation: 4.5 partial threated screws, 6.5 partial or fully threated screws. To perform a successful minimally invasive operative treatment some surgical instruments are mandatory: lamina spreader, various types of bone distractors, Steinmann pins, different size elevators, heel bone press, periarticular reduction forceps (9). Proper positioning of the patient on the operative table and no movement of patient or image intensifier during surgery are mandatory. Preoperative adjustment of the three X-ray views - later- 
al, axial and Broden view is crucial for the success of the procedure (9).

\section{CASE I}

A 55-year-old female presented with extreme beak fracture after falling from a chair (Fig. 1). This kind of calcaneal fracture should be operated on emergently because of marked disruption of the posterior soft tissues, the pressure from within on the posterior skin will soon cause it to break down and convert it into an open fracture. Skin necrosis may occur in a few hours if reduction is not performed. After reduction and fixation was performed with two 4.5 cannulated screws and one $6.5 \mathrm{~mm}$ fully threated cannulated screw (Fig. 2).

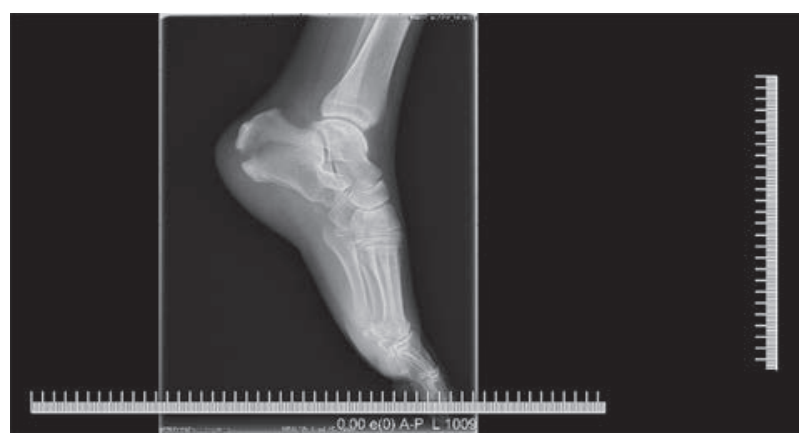

Fig. 1. Preoperative X-ray image

\section{CASE II}

The patient was a 51-year-old male, heavy smoker for more than twenty years, with joint-depression type fracture, preoperative X-ray and CT scan with 3D reconstruction (Fig. 3. A, B, C). There was severe calcinosis of vessels around the ankle. Reduction was performed with Hintermann distractor and lamina spreader; fixation was achieved with 4 cannulated screws (Fig. 4. A, B).

\section{CASE III}

A male patient at the age of 44 , with diabetes mellitus type I of more than 20 years, presented with tongue type fracture of the calcaneus. Preoperative X-ray and CT scan were done (Fig. 5. A, B, C). Reduction was achieved by using a Steinmann pin as joystick technique and fixation was done with 4 cannulated screws (Fig. 6. A, B, C).

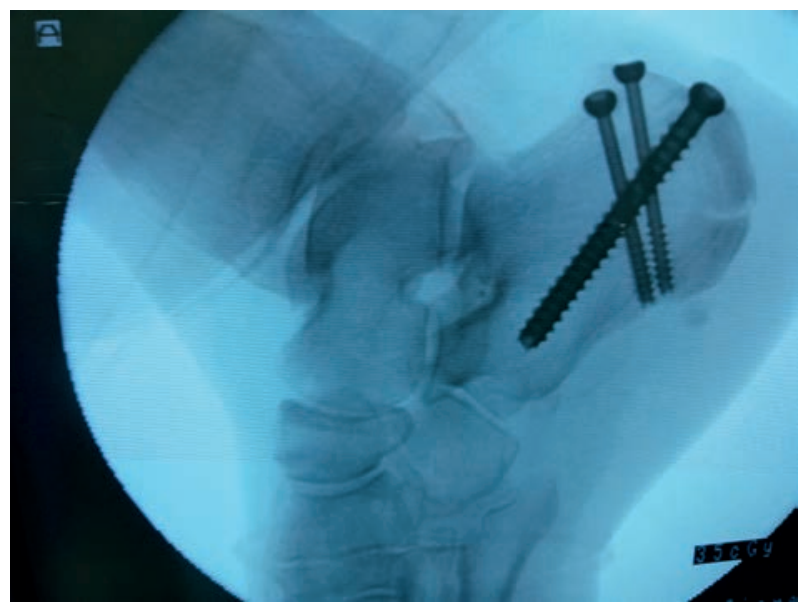

Fig. 2. Postoperative X-ray image
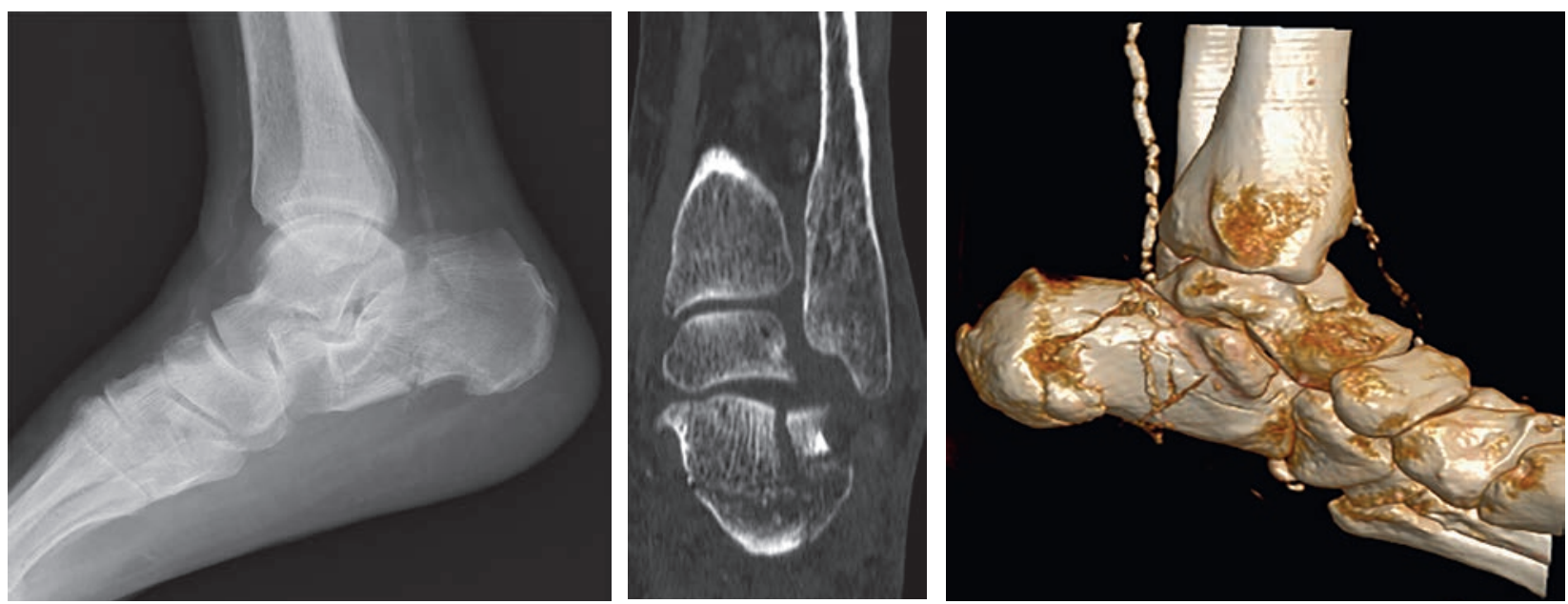

Fig. 3. A, B, C. Preoperative X-ray and CT scan with $3 D$ reconstruction, joint-depression type fracture 
Cannulated Screw Fixation for Dislocated Fractures of the Calcaneus
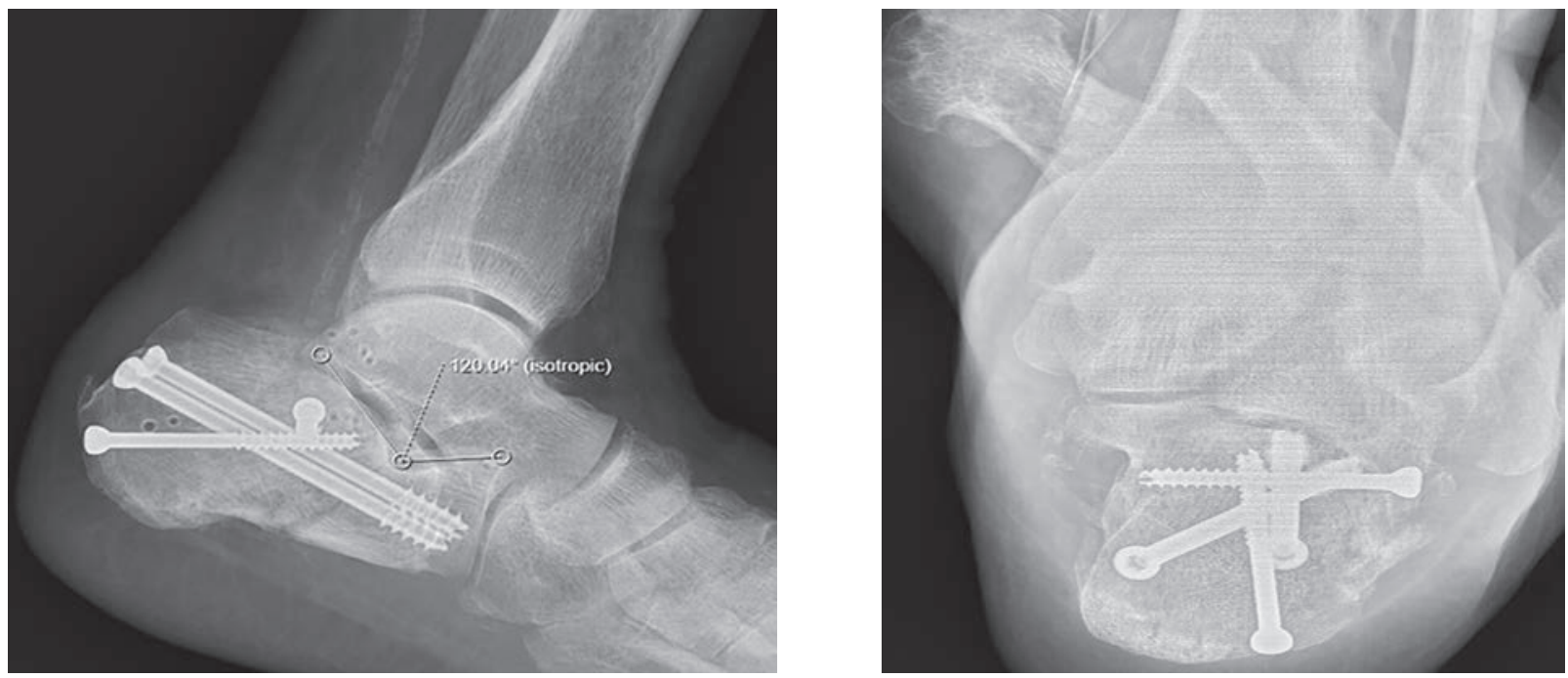

Fig. 4. A, B. Postoperative $X$-ray image (lateral and axial view)
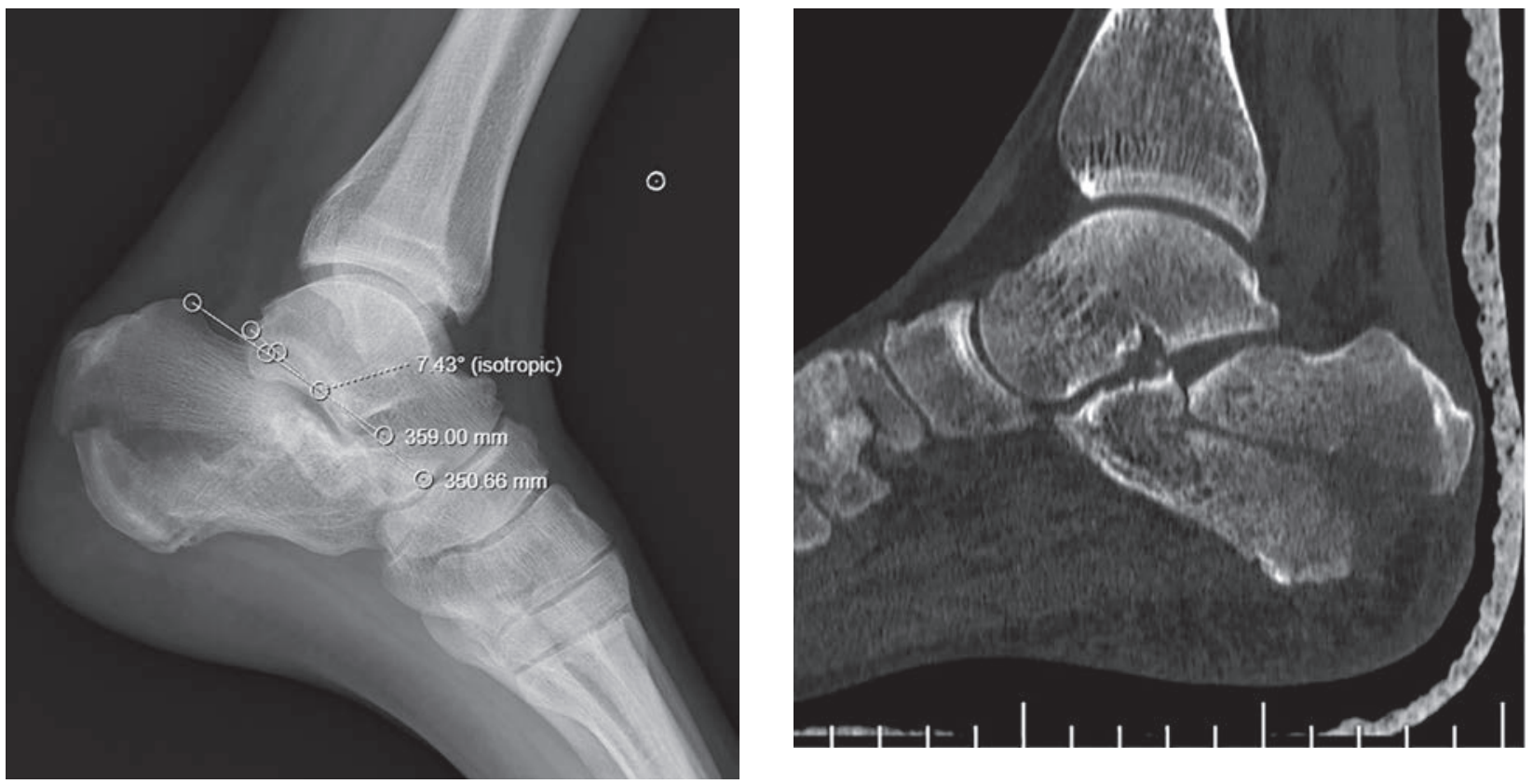

Fig. 5. A, B, C. Preoperative X-ray and CT scan, tongue type fracture
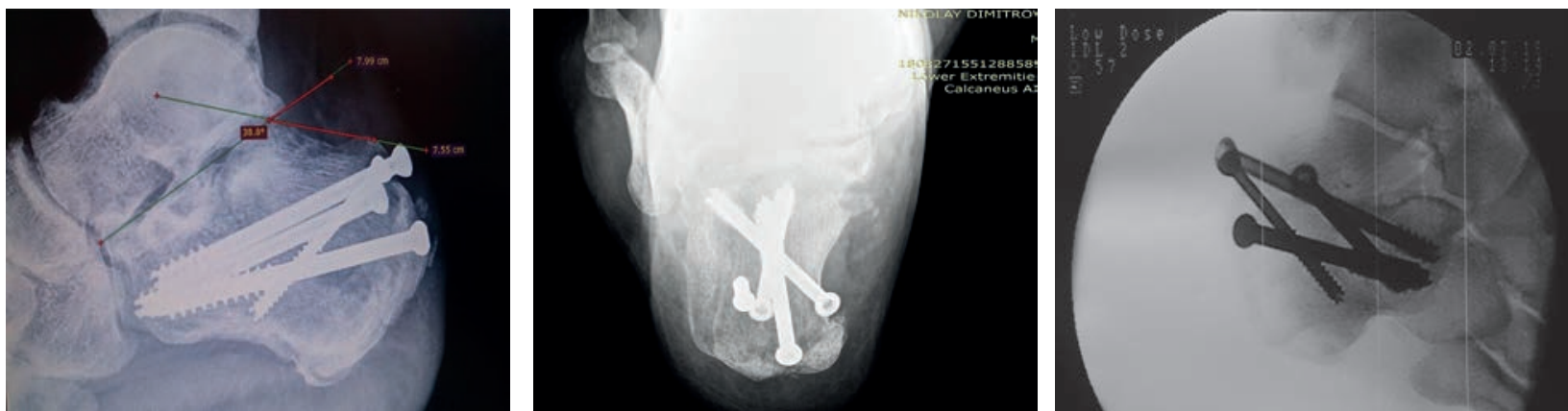

Fig. 6 A, B, C. Postoperative $X$-ray picture (lateral, axial view and Broden view) 


\section{RESULTS}

The study included 18 men and 2 women, fixation was achieved by different types of cannulated screws. Evaluation of the results were made by using the Maryland Foot Score. Eleven patients had excellent result (55\%), 7 patients - good result (35\%), 2 patients - satisfactory one (10\%), and 0 patients bad result (0\%). Satisfaction from the treatment was graded using the Visual Analogue Scale (VAS) score with a mean score of 7.5. The average time from admission to surgery was 4 days, the mean duration of surgery was 58 minutes. All patients were followed up and all fractures healed. There were no cases with wound healing problems - infection or skin necrosis. In the postoperative period, the regular changes of the dressings were mandatory and intensive cryotherapy was performed to reduce swelling, no cast immobilization was used and gradual early active range of motion was encouraged without weight bearing. Mean hospitalization time was 5 days. Earlier implant removal due to prominent hardware causing peroneal impingement and tendinitis was performed in one patient. Transient paresthesia of nervus suralis was recorded in one patient, which resolved spontaneously in a few weeks.

The reduction was evaluated by measurement of the angle of Gissane, Bohler and Preis pre- and postoperatively on lateral and axial views. Unfortunately, no postoperative CT scan could be performed and we evaluated the short follow-up period in the study. Due to strict control and patient compliance there was no loss on reduction in the follow-up period on conventional X-ray in this study.

\section{DISCUSSION}

The main factors that affect the prognosis after surgical treatment of calcaneus fractures are reconstruction of the congruence of the articular surfaces and restoration of bone alignment and morphology, thus preserving the normal biomechanics of the foot. Therefore, stable and secure fixation of the fracture is mandatory. Adequate fixation of these fractures is still a hot topic in clinical and biomechanical studies.

Despite technical evolution of medicine and the use of new implants regarding calcaneus fractures smaller plates, locking plates with angular stability, open reduction and internal fixation techniques still have been reported to have a high percentage of soft tissue complications from $10 \%$ to $25 \%$. Moreover in patients with high risk like diabetics, smokers, with vascular disease or immune deficiency and in open fractures the complication rate further increases.

After open procedures the functional outcome may be compromised due to severe soft tissue dissection and striping. The use of cannulated screws through stab incisions after adequate reduction of the fracture provides the opportunity to avoid a lot of these complications. Even more this preserves the soft tissues, allows rapid bone consolidation and faster rehabilitation $(10,11)$. For cannulated screws fixation (minimally invasive techniques) early surgery is mandatory, mobilization of the displaced fragments is easier and better reduction can be achieved. Early surgery leads to a decrease of the swelling and avoidance of fracture blisters.

Due to the unique bone morphology accurate screw placement is a challenge. The entry points, screw paths trajectories and screw lengths depends on the type of the fracture and the direction of dislocated fragments $(12,13,14)$. The aim is to place the screws in such way to create a support in the dense spongy bone and to ensure stable fixation of the posterior facet. The points that can ensure stable screw fixation are tuber calcanei, anterior process, sustentaculum tali and subchondral bone of the posterior facet. Fully treated cannulated screws are perfect to be used to hold and restore heel length and height after proper reduction. Usually $4.5 \mathrm{~mm}$ screws are used to fix the posterior facet, they could be inserted transversely to induce compression between fragments or screws can be guided from tuber calcanei to the subchondral bone of the posterior facet depending on the direction of the fracture lines.

The heads of the screws should be buried below the level of the cortex to minimizes the chance of irritation and possible discomfort. Incorrect placement of screws may lead to severe complications - intraarticular penetration and arthritis, pain, swelling, tendon (peroneal or flexor hallucis longus) impingement and nerve injuries (paresthesia, tarsal tunnel syndrome).

In the literature there are a lot of studies regarding safe screw localization. Based on 3D bone model in a radio-anatomical study Phisitkul P et al. (15) investigated the best trajectory for sustentacular screw 
placement. The conclusions were that the sustentacular screw should be approximately $40 \mathrm{~mm}$ in length, entry point should start $15 \mathrm{~mm}$ below the posterior facet measured perpendicular to the joint line, the anteversion angle increases at each position along the posterolateral facet from 6 to 36 degrees from anterior to posterior and the subtalar convergence angle should be 20 degrees.

In a biomechanical study, Carr et al. (16) investigated the effect of plate size on the fixation strength and their conclusion was that the reduction with bone contact is important (spongy bone), not the plate size, so a reposition should achieve the best posterior facet reduction. In a cadaveric biomechanical study by Smerek et. al. (17), it was emphasized that percutaneous screw fixation of calcaneus fractures may provide a strength similar to that of plate fixation. Different implants can be used for fracture synthesis and the goal is to achieve enough stability to promote early active range of motion, without weight bearing and redisplacement of the fragments $(18,19)$.

Optimal reduction of the articular surface could be improved by utilizing the sinus tarsi approach. In addition, arthroscopy of the subtalar joint is an excellent option for joint reduction. Proper screw placement could be facilitated if the surgeon uses modern CT scan reconstruction intraoperatively.

\section{CONCLUSION}

Cannulated screw fixation in dislocated calcaneus fractures is a technically demanding procedure. Good spatial orientation, surgical experience and preoperative planning (CT scan in all planes and $\mathrm{X}$-ray images) are mandatory (20). Patient selection, close follow up and compliance are crucial for optimal functional results. Cannulated screw fixation in calcaneus fractures is an effective surgical option with good clinical result, short hospitalization time, minimal blood loss and soft tissue dissection with reduced risk of complications.

\section{REFERENCES}

1. Palmer I. The mechanism and treatment of fractures of the calcaneus. Open reduction with use of cancellous grafts. J Bone Joint Surg Am. 1948; 30A(1):2-8.

2. Buckley R, Tough S, McCormack R, Pate G, Leighton R, Petrie D, et al. Operative compared with nonoperative treatment of displaced intraarticular calcaneal fractures: a prospective, randomized, controlled multicenter trial. J Bone Joint Surg Am. 2002; 84(10):1733-44.

3. Benirske SK, Sangeorzgan BJ. Extensive intraarticular fractures of the foot. Surgical management of calcaneal fractures. Clin. Orthop. 1993; 292:128-34.

4. Forgon M: Closed reduction and percutaneous osteosynthesis: Technique and results in 265 calcaneal fractures. In: Tscherne H, Schatzker J, eds. Major fractures of the pilon, the talus, and the calcaneus. New York: Springer- Verlag; 1993. p. 207-13.

5. Stulik J, Stehlik J, Rysavy M, Wozniak A. Minimally-invasive treatment of intraarticular fractures of the calcaneum. J Bone Joint Surg Br. 2006;88(12):1634-41. doi: 10.1302/0301-620X.88B12.17379.

6. Sanders R, Fortin P, Dipasquale T. Operative treatment of 120 displaced intraarticular calcaneal fractures. Results using a prognostic computed tomography scan classification. Clin Orthop Relat Res. 1993;(290):87-95.

7. Bohler L. Diagnosis, pathology and treatment of fractures of the os calcis, J Bone Joint Surg Am. 1931; 13(1):75-89.

8. Essex - Lopresti P. The mechanism, reduction technique and results in fractures of the os calcis; British J Surg. 1952; 39(157):395-419.

9. Broden B. Roentgen examination of the subtaliod joint in fractures of the calcaneus. Acta Radiol. 1949;31(1):85-91.

10. Schepers T, Schipper IB, Vogels LM, Ginai AZ, Mulder PG, Heetveld MJ, et al. Percutaneous treatment of displaced intra-articular calcaneal fractures. J Orthop Sci. 2007; 12(1):22-7. doi: 10.1007/s00776-006-1076-z.

11. Patel N, Bhavsar N, Lil N. Percutaneous fixation of Calcaneum fractures. GCSMC J Med Sci. 2014;3(1):31-4.

12. Qiang $M$, Chen $Y$, Zhang K, Li H, Dai H. Effect of sustentaculum screw placement on outcomes of intraarticular calcaneal fracture osteosynthesis: A prospective cohort study using 3D CT. Int J Surg. 2015;19:72-7. doi: 10.1016/j.ijsu.2015.05.011.

13. Wang C, Huang D, Ma X, Wang X, Huang J, Zhang C, et al. Sustentacular screw placement with guidance during ORIF of calcaneal fracture: an 
anatomical specimen study. J Orthop Surg Res. 2017;12(1):78. doi: 10.1186/s13018-017-0580-5.

14. Labronici1 PJ, Pereira DN, Pilar M, Franco JS, Serra MD, Cohen JC, et al. Safe localization for placement of percutaneous pins in the calcaneus. Rev Bras Ortop. 2012;47(4):455-9. doi: 10.1016/ S2255-4971(15)30128-2

15. Phisitkul P, Sullivan J, Goetz J, Marsh J. Maximizing safety in screw placement for posterior facet fixation in calcaneus fractures: A cadaveric radio-anatomical study. Foot Ankle Int. 2013;34(9):1279-85. doi: 10.1177/1071100713487182.

16. Carr J, Tigges R, Wayne J, Mark E. Internal fixation of experimental intraarticular calcaneal fractures: A biomechanical analysis of two fixation methods. J Orthop Trauma. 1997;11(6):425-8; discussion 428-9.

17. Smerek JP, Kadakia A, Belkoff SM, Knight TA, Myerson MS, Jeng CL. Percutaneous screw configuration versus perimeter plating of calcaneus fractures: A cadaver study. Foot Ankle Int. 2008;29(9):931-5. doi: 10.3113/FAI.2008.0931.
18. Ming N, Mei J, Lil K, Niu W, Zhang M. The primary stability of different implants for intra-articular calcaneal fractures: an in vitro study. Biomed Eng Online. 2018;17(1):50. doi: 10.1186/s12938-018-0484-6.

19. Rausch S, Klos K, Wolf U, Gras M, Simons P, Gueorguiev B, et al. A biomechanical comparison of fixed angle locking compression plate osteosynthesis and cement augmented screw osteosynthesis in the management of intra articular calcaneal fractures; Springer-Verlag Berlin Heidelberg; 2014.

20. Gitajn IL, Toussaint RJ, Kwon JY. Assessing accuracy of sustentaculum screw placement during calcaneal fixation. Foot Ankle Int. 2013;34(2):2826. doi: 10.1177/1071100712467617. 